\title{
Essais
}

ESSAIS

Revue interdisciplinaire d'Humanités

$10 \mid 2016$

Faire-valoir et seconds couteaux

\section{Personnage secondaire ou second héros : la place paradoxale de Sam dans Le Seigneur des Anneaux}

\section{Antoine Paris}

\section{(2) OpenEdition}

1 Journals

Édition électronique

URL : http://journals.openedition.org/essais/3848

DOI : 10.4000/essais.3848

ISSN : 2276-0970

Éditeur

École doctorale Montaigne Humanités

Édition imprimée

Date de publication : 15 septembre 2016

Pagination : 69-80

ISBN : 978-2-9544269-9-0

ISSN : 2417-4211

Référence électronique

Antoine Paris, «Personnage secondaire ou second héros : la place paradoxale de Sam dans Le Seigneur des Anneaux », Essais [En ligne], 10 | 2016, mis en ligne le 15 octobre 2020, consulté le 21 octobre 2020. URL : http://journals.openedition.org/essais/3848 ; DOI : https://doi.org/10.4000/ essais.3848 


\title{
Personnage secondaire ou second héros : la place paradoxale de Sam dans Le Seigneur des Anneaux
}

\author{
Antoine Paris
}

"Assurément, Sam est le personnage le plus minutieusement dépeint, le successeur du Bilbo du premier livre ${ }^{1}$, le Hobbit authentique. Frodo n'est pas aussi intéressant, parce qu'il se doit d'être digne et possède (pour ainsi dire) une vocation. Le livre s'achèvera sans doute sur Sam. Frodo va naturellement devenir trop ennobli et raffiné par la réalisation de cette Quête grandiose, et il passera à l'Ouest avec toutes les grandes figures ; mais $S$. va se fixer en Comté, avec ses jardins et ses auberges. " Ces lignes extraites d'une lettre adressée par John Ronald Reuel Tolkien à son fils Christopher comportent plusieurs éléments qui, me semble-t-il, posent le problème des places respectives de Frodo et Sam à l'intérieur de son œuvre la plus connue, Le Seigneur des Anneaux².

Le terme même de "Quête " employé par Tolkien peut inciter à analyser les relations narratives entre les deux personnages en fonction du schéma actantiel de Greimas ${ }^{3}$, où la " quête » est centrale. Le terme apparaît également dans le roman lui-même, notamment dans les paroles de Frodo, qui vient de détruire l'Anneau : " "Yes", said Frodo. "But do you remember Gandalf’s words: Even Gollum may have something yet to do? But for him, Sam, I could not have destroyed the Ring. The Quest would have been in vain, even at the bitter end. So let us forgive him! For the Quest is achieved, and now all is over. " (VI, $\left.3^{4}\right)$ Frodo, dans les termes de Greimas, serait le "sujet ",

1 The Hobbit, premier livre publié par John Ronald Reuel Tolkien, en 1937 par Allen \& Unwin.

2 «Extrait d'une lettre à Christopher Tolkien » datée du 24 décembre 1944.354 lettres de Tolkien ont été sélectionnées et éditées par Humphrey Carpenter dans The letters of J.R.R. Tolkien (HarperCollinsPublishers, 1981). Je cite la traduction française de Delphine Martin et Vincent Ferré (J.R.R. Tolkien, Lettres, Christian Bourgeois Éditeur, 2005). Il s'agit de la lettre 93, figurant à la p. 106 de l'édition anglaise et à la p. 155 de la traduction en français.

3 Greimas, Algirdas Julien, Sémantique structurale : recherche et méthode, Larousse, 1966.

4 J'indique en chiffres romains le livre, suivi du numéro du chapitre. (Selon une habitude qui s'est perpétuée, Le Seigneur des Anneaux est le plus souvent édité en trois volumes, chacun regroupant deux livres.) 
menant la "quête " qui constitue l'intrigue principale du roman, à savoir la destruction de l'Anneau unique, seul acte capable de vaincre le Seigneur du Mordor, Sauron. Mais étrangement, ce statut même rendrait Frodo moins " intéressant » et expliquerait qu'il soit moins " minutieusement dépeint ». En termes d'intérêt, la première place serait ainsi occupée par Sam, pourtant simple "adjuvant " dans le schéma narratif. De la même façon, paradoxalement, c'est en raison de la quête qu'il a menée et qui lui confère son statut de " héros » que Frodo devrait, à la fin du roman, céder la place et le dernier mot à Sam. Signalons toutefois une limite à l'application du schéma actantiel dans le cas du Seigneur des Anneaux, due à une caractéristique du monde narratif de Tolkien. Comme l'indique la référence à Gollum dans la citation précédente, la quête n'a pas été menée à son terme par Frodo. Parvenu dans le Sammath Naur où il pourrait enfin jeter l'Anneau dans le feu qui y brûle, Frodo cède au pouvoir de l'objet maléfique et refuse de le détruire. C'est seulement parce que Gollum s'empare alors par violence de l'Anneau avant de tomber dans la fournaise que l'artefact de Sauron peut enfin disparaître. Ainsi, d'une certaine manière, la quête est réussie malgré tous les personnages, ce qui ne peut qu'amener à relativiser l'idée de "sujet » ou de " héros ».

La lettre de Tolkien comporte un dernier paradoxe, concernant la relation entre Sam et Bilbo : alors que Frodo est, dans le récit, le neveu de Bilbo et à ce titre son héritier, Sam serait, d'une autre façon peut-être, « le successeur du Bilbo du premier livre ». Ainsi, malgré le fait que Sam ne soit pas le responsable de la "Quête grandiose ", il y aurait comme un passage de témoin entre le héros du premier livre de Tolkien et lui.

Après avoir étudié ce qui pourrait faire de Sam un second couteau du Seigneur des Anneaux, je m'intéresserai à un chapitre décisif concernant sa place en tant que personnage. À la fin du livre IV, Frodo semblant mort, Sam hésite à prendre l'Anneau pour poursuivre la "Quête ", ce qui ferait de lui le personnage principal de l'intrigue. Enfin, je me demanderai si le statut de Sam comme second couteau ne peut pas être éclairée par la dimension métalittéraire du Seigneur des Anneaux.

Lorsque Gandalf présente à Frodo la quête qui sera la sienne, le magicien évoque la possibilité pour lui de choisir un compagnon, en des termes qui seraient presque une définition du rôle d'un adjuvant : «But I don't think you need to go alone. Not if you know of anyone you can trust, and who would be willing to go by your side -and that you would be willing to go by your side- and that you would be willing to take into unknown perils. " (I, 2) Sam sera la figure parfaite de ce compagnon recherché.

Par plusieurs aspects, Sam Gamgee est inférieur à Frodo, que ce soit socialement ou, pourrait-on dire, en termes de registre littéraire. La chronologie présente dans l'appendice B indique que, né en l'an 2980 du "Troisième Âge ", il est de douze ans plus jeune que Frodo, dont la naissance est située en 
29685. Une autre manifestation de l'infériorité de Sam serait sa maladresse. En Lorien, il éprouve de grandes difficultés à traverser un pont provisoire en corde : "Sam shuffled along, clutching hard, and looking down into the pale eddying water as if it was a chasm in the mountains. " (II, 6) Par ailleurs, Sam se caractérise par une certaine simplicité d'esprit. Ce serait le sens de l'étymologie anglaise de son nom, selon une lettre de Tolkien : "Sam est une abréviation non de Samuel mais de Samwise [Samsagace], équivalent au vieil anglais de half-wit [simplet]. " ${ }^{6}$ Cette naïveté fait de Sam un gaffeur potentiel, comme lorsqu'il révèle à Faramir l'objet de la quête de son Maître, que ce dernier avait tout fait pour maintenir secret (IV, 5). Par cette caractéristique, Sam semble se rattacher à un registre littéraire différent de celui auquel appartiennent Frodo et la plupart des personnages du récit, le registre comique, en décalage avec ces figures héroïques. Ainsi, irrité par les soupçons de Faramir à l'égard de Frodo, Sam "planted himself squarely in front of Faramir, his hands on his hips, and a look on his face as if he was addressing a young hobbit who had offered him what he called "sauce" when questioned about visits to the orchard. " (IV, 5). Outre le choc entre l'univers épique de Faramir et le monde villageois de Sam, la citation d'un idiolecte du serviteur de Frodo (" sauce » - "balivernes » en français) est en total décalage avec la situation. Il rappelle en outre que Frodo et Sam appartiennent à deux classes sociales différentes : avant que la Quête ne commence, Sam est le jardinier de Bilbo et de son oncle ${ }^{7}$.

Ses liens avec Frodo sont ceux d'un serviteur ("servant ") à son maître («master ») selon deux termes fréquemment employés. Dans son article de 2004, Mark T. Hooker a proposé de voir dans cette relation un reflet de celle qui unissait lors de la Première guerre mondiale un officier anglais et son "batman $»^{8}$. La comparaison qu'il propose avec certains romans de guerre décrivant les actions de ces serviteurs militaires est convaincante et d'autant plus pertinente que Tolkien lui-même compare Sam aux «soldats anglais, aux combattants et aux batmen que j'ai connus pendant la guerre de 1914 " $^{9}$ Le batman a d'abord pour tâcher de s'occuper des bagages de l'officier auquel il est attaché, comme l'indique son nom, formé sur le français " bât $»^{10}$. Les préparatifs au départ de Fondcombe montrent toute l'importance que Sam accorde au service zélé de Frodo quant à

5 Appendice B, p. 1064.

6 "Lettre à Christopher Tolkien ", portant le numéro 72 dans le recueil déjà mentionné (p. 83 dans l'édition anglaise, p. 124-125 dans la traduction française).

7 Cette différence de classe sociale est notamment étudiée par Mark T. Hooker, qui y voit le reflet de la société anglaise de l'époque de la Première guerre mondiale. (Hooker, Mark T., "Frodo’s batman ", Tolkien Studies 1.1, 2004, p. 125-136, p. 131 à 133 pour ce point précis.

8 Hooker, Mark T., art. cit.

9 Cité dans Carpenter, Humphrey, J.R.R. Tolkien: a biography, George Allen \& Unwin, 1977, p. 91.

10 Hooker, Mark T., art. cit., p. 125. 
ce point précis : il rassemble « various small belongings of his master's that Frodo had forgotten and Sam had stowed to bring them out in triumph when they were called for. " (II, 3) Sam est aussi celui qui s'occupe de préparer les repas pour son maître, comme lors de l'épisode relaté dans le chapitre « Of herbs and stewed rabbits " (IV, 4). Ceci pourrait encore être caractéristique de la mémoire des tranchées: Anna Smol cite le cas de "men taking on traditionally female domestic and nurturing role in the First World War ${ }^{11}$ et Mark T. Hooker rapporte plusieurs anecdotes concernant des batmen improvisant des repas pour leurs officiers, même au milieu de villages dévastés ${ }^{12}$. Tout comme les serviteurs d'officiers, Sam témoigne d'une fidélité obstinée envers son maître. Au conseil d'Elrond il lui-même insiste pour accompagner Frodo (II, 2). Voyant dans le miroir de Galadriel la possible ruine de la Comté si chère à son cœur, mais aussi de la maison de son propre père, il fait le choix de suivre son maitre plutôt que de rentrer chez lui (II, 7). Lorsqu'à la fin du livre II, Aragorn propose de choisir parmi les membres de la Compagnie de l'Anneau des alliés pour soutenir Frodo dans son voyage au Mordor, cette liste comprend en premier lieu Sam " who could not bear it otherwise " (II, 10). Enfin, son maitre ayant décidé de partir seul au Mordor, il manque de se noyer en se lançant dans l'eau à sa suite (II, 10). Cette importance du personnage en tant qu'adjuvant apparaît surtout à partir du livre IV, lorsque Frodo commence son voyage avec Sam à travers les terres désolées qui le séparent de la Crevasse du Destin. Son aide prend à la fois la forme d'une assistance matérielle et concrète mais se manifeste aussi par de nombreux gestes de tendresse, tels que celui par lequel il répond à une inquiétude de son maitre dans le chapitre 2 du livre IV : "Sam nodded silently. He took his master's hand and bent over it. He did not kiss it, though his tears fell on " De tels gestes ont pu déconcerter et amener à voir Frodo et Sam comme des figures homosexuelles, d'autant plus que le verbe " aimer " est plusieurs fois employé par Sam pour décrire ses sentiments pour Frodo ("I love him », peut-on lire en IV, 4). Anna Smol propose une interprétation très fine de cette question. À nouveau, le souvenir de la Première guerre mondiale a pu jouer un rôle ici et l'auteure cite à ce sujet Santanu Das, ${ }^{13}$ qui évoque une « largely nongenital tactile tenderness ${ }^{14}$ parmi les soldats des tranchées, suscitée notamment par la proximité des dangers et de la mort. Plutôt que d'" homosexualité », il faudrait parler d'une " homosocial relationship ${ }^{15}$ qui, de fait, constitue une transgression des codifications de genre traditionnelles. Cet aspect transgressif

11 Smol, Anna, " "Oh... oh... Frodo!": Readings of male intimacy in The Lord of the Rings ", Modern Fiction Studies, vol. 50, n 4, hiver 2004, p. 949-949, p. 954.

12 Hooker, Mark T., art. cit., p. 126 notamment.

13 Smol, Anna, art. cit., p. 955.

14 Das, Santanu. " "Kiss me, Hardy": Intimacy, gender, and gesture in World War I trench literature. " Modernism/Modernity 9, 2002, p. 51-74 (p. 52).

15 Smol, Anna, art. cit., p. 956. 
de la relation entre les deux hobbits, socialement parlant, apparait d'ailleurs, me semble-t-il, lors du retour dans la Comté lorsque Sam, marié, emménage avec son épouse auprès de Frodo (VI, 9). Il faudra d'une certaine manière le départ de son compagnon vers l'Ouest pour que Sam commence une vie de famille traditionnelle. La relation entre les deux hobbits pourrait enfin donner lieu à une lecture religieuse. Par plusieurs aspects, Frodo est assimilé au Christ, notamment par le fait qu'il « porte » le fardeau de l'Anneau, comme le Christ porte sa croix. Dans cette lecture, Sam pourrait correspondre à Simon de Cyrène, aidant Jésus à porter l'instrument de son supplice : «I can't carry it for you, but I can carry you and it as well. ", déclare Sam à Frodo en VI, 3, lors de l'ascension de Mount Doom.

Plusieurs modèles pourraient ainsi permettre de décrire la relation entre Frodo et Sam, dont chacune paraît pertinente et en même temps insuffisante : outre la lecture religieuse, l'interprétation homosociale et les parallèles avec la Première guerre mondiale, il serait encore possible de voir dans les liens entre les deux hobbits ceux d'un écuyer et d'un chevalier, tels qu'ils se présentent dans la littérature médiévale. Il me semble que deux autres éléments peuvent être notés, qui correspondent à l'originalité de Sam en tant que personnage secondaire.

Sam est le jardinier de Bilbo et de son neveu Frodo. Cette information est la première qui nous est donnée à son sujet, en même temps que sa filiation : " old Ham Gamgee, commonly known as the Gaffer (...) had tended the garden at Bag End for forty years (...) Now that he was himself growing old and stiff in the joints, the job was mainly carried on by his youngest son, Sam Gamgee. » (I, 1) Cet aspect de la personnalité de Sam explique que, même au milieu de leur périple vers le Mordor, traversant l'Ithilien, il observe les plantes inconnues qui s'y trouvent, sans souci du péril (IV, 4). Le cadeau que lui offre Galadriel est parfaitement adapté à ses amours : " "For you little gardener and lover of trees", she said to Sam, "I have only a small gift." She put into his hand a little box of plain grey wood, unadorned save for a single silver rune upon the lid. "Here is set G for Galadriel", she said; "but also it may stand for garden in your tongue. In this box there is earth from my orchard, and such blessing as Galadriel has still to bestow is upon it”. " (II, 8) Grâce à cette terre bénie, Sam pourra à la fin du roman repeupler d'arbres magnifiques toute la Comté dévastée par les méfaits de Saruman (VI, 9). Ce rapport particulier à la nature trouve son prolongement dans l'amour de Sam pour son pays natal. Ainsi il a un rôle de premier plan dans les combats pour libérer la Comté de l'emprise du magicien malfaisant, alors que justement Frodo apparaît comme plus effacé lors de ces événements (VI, 9). Son nom même, selon une étymologie imaginaire présente dans un des appendices, correspondrait à cette vie de village : "Gamgee. According to family tradition, set out in the Red Book, the surname Galbasi, or in reduced form Galpsi, came from the village of Galabas (...) » (Appendice F). 
Par ailleurs, il me semble important de souligner que le départ de Sam à la suite de Frodo n'est pas dû à une décision de sa part. Au moment où Gandalf révèle à Frodo la vérité sur l'Anneau et l'encourage à quitter la Comté au plus vite avec l'objet maléfique, il surprend Sam qui les écoutait à la fenêtre. (I, 2) C'est pour cette raison que Gandalf le désigne comme serviteur de Frodo dans son voyage : "I have thought of something better than that. Something to shut your mouth, and punish you properly for listening. You shall go away with Mr. Frodo! » (I, 2) Â nouveau, me semble-t-il, l’idée de schéma actantiel et les notions de "sujet " et d'" adjuvant " doivent être nuancées. Comme dans la scène finale de la destruction de l'Anneau, ce sont les circonstances - ou une forme de providence - plutôt que leurs choix et liberté qui guident les personnages. Sam n'a pas fait le choix d'accompagner Frodo dans sa quête, pas plus que ce dernier n'a choisi d'en être le sujet.

Il me semble que cette idée d'absence de choix initial pourrait éclairer le chapitre final du livre IV, justement intitué " The choices of Master Samwise ». Trahis par leur guide Gollum, Sam et Frodo sont attaqués par la terrifiante araignée géante Shelob. Sam retrouve son maitre allongé à terre, sans respiration ni battement de cœur. Le présumant mort il doit choisir entre rester défendre son corps ou prendre l'Anneau suspendu à son cou pour poursuivre la Quête. En d'autres termes, Sam aurait ainsi à choisir entre rester un second couteau ou devenir le nouveau personnage principal. Je suivrai ici la narration du passage en m'interrogeant d'abord sur la décision de Sam d'assumer la Quête à la place de son maître, puis sur son revirement. Enfin, je me demanderai dans quelle mesure le rapport à l'acte de choisir lui-même pourrait permettre d'éclairer le personnage de Sam en tant que personnage secondaire.

Après la supposée mort de Frodo, Sam prend son épée Dard et la fiole que le héros avait reçue de Galadriel. De cette façon un passage de relais semble s'opérer. Mais seul l'Anneau pourrait permettre de constituer Sam en personnage principal. "Frodo had died and laid aside the Quest. » (IV, 10) La question sera de savoir si Sam décide de la poursuivre à la place de son maître ou non. L'enjeu narratif derrière ce choix apparait, me semble-t-il, dans le monologue qui suit.

\footnotetext{
" "What am I to do then?" he cried again, and now he seemed plainly to know the hard answer: see it through. Another lonely journey, and the worst.

"What? Me, alone, go to the Crack of Doom and all?" He quailed still, but the resolve grew. "What? Me take the Ring from him? The Council gave it to him."

"But the answer came at once: And the Council gave him companions, so that the errand should not fail. And you are the last of all the Company. The errand must not fail."

"I wish I wasn't the last", he groaned. "I wish old Gandalf was here, or somebody. Why am I left all alone to make up my mind? I'm sure to go wrong. And it's not for me to go taking the Ring, putting myself forward."

"But you haven't put yourself forward; you've been put forward. And as for not being the right and proper person, why, Mr. Frodo wasn't, as you might say, nor Mr. Bilbo. They didn't choose themselves.” "
} 
L'expression "put oneself forward " exprime le fait de se porter volontaire. Mais il me semble qu'elle pourrait aussi signifier "se mettre en avant ". Le choix que doit faire Sam oppose en effet deux possibilités : rester derrière Frodo, même s'il ne s'agit plus que de protéger sa dépouille, ou bien choisir de poursuivre la quête et ainsi passer de l'arrière-plan à l'avant-scène. C'est l'un des sens que pourrait revêtir la mention de Frodo, mais aussi de Bilbo dans la dernière phrase. Bilbo était le personnage principal du Hobbit, Frodo fut celui du Seigneur des Anneaux jusqu'à ce chapitre où la mort semble l'avoir emporté. Sam pourrait être à leur suite le héros d'une seconde partie du Seigneur des Anneaux qui commencerait maintenant. Considérant l'intérêt général - que le monde est perdu si l'Anneau tombe aux mains de l'ennemi - c'est ce choix que commence par faire Sam.

" "Let me see now: if we're found here, or Mr. Frodo's found, and that Thing's on him, well, the Enemy will get it. And that's the end of all of us, of Lorien, and Rivendell, and the Shire and all. And there's no time to lose, or it'll be the end anyway. The war's begun, and more than likely things are all going the Enemy's way already. No chance to go back with It and get advice or permission. No, it's sit here till they come and kill me over master's body, and gets It; or take It and go." He drew a deep breath. "Then take It, it is!" "

Mais Sam revient aussitôt sur cette décision lorsqu'une compagnie d'Orcs surgit et qu'ils découvrent le corps inanimé de son maitre.

«I wonder if any song will ever mention it: How Samwise fell in the High Pass and made a wall of bodies round his master. No, no song. Of course not, for the Ring'll be found, and there'll be no more songs. I can't help it. My place is by Mr. Frodo. They must understand that -Elrond and the Council, and the great Lords and Ladies with all their wisdom. Their plans have gone wrong. I can't be their Ring-bearer. Not without Mr. Frodo.»

Au moment où le corps de son maître est menacé, la considération d'un intérêt général n'a plus de sens. Sam évoque à nouveau la menace qui pèse sur toute la Terre du Milieu, à travers l'évocation des chansons qui ne seront plus, mais ce futur de destruction générale est désormais pris comme une fatalité : il ne pourra en être autrement car " la place " de Sam est " auprès de M. Frodo". Par l'expression de "Ring-bearer ", niée, Sam rejette la possibilité d'être le porteur de l'Anneau et ainsi le nouveau personnage principal. Bien qu'elle se trouve a posteriori justifiée par la nouvelle que Sam apprendra par la suite - que Frodo n'est pas mort mais qu'il a seulement été paralysé par le venin de Shelob cette décision apparaît comme suicidaire, et pour lui-même, et pour le monde : plutôt mourir sur le corps de son maître et faire périr toute la Terre du Milieu avec lui que d'abandonner Frodo à l'ennemi, fût-ce à l'état de cadavre.

Mais s'agit-il vraiment d'une décision ? Sam, au moment de choisir l'Anneau, est persuadé que quoi qu'il arrive il fera un mauvais choix : "Ah well, I must make up my own mind. I will make it up. But I'll be sure to go 
wrong: that'd be Sam Gamgee all over. » De fait, une fois que ce choix est fait, Sam ressent aussitôt que l'option choisie est " against the grain of his nature ". Une idée similaire est exprimée à la fin du chapitre : "You fool, he isn't dead, and your heart knew it. Don't trust your head, Samwise, it is not the best part of you. " (IV, 10) Il y aurait ici comme l'expression par Sam de la conscience de sa propre naïveté, mais en même temps, il pourrait apparaître comme la seule figure dans Le Seigneur des Anneaux à savoir qu'au fond, les choix sont un leurre, que les événements sont guidés par une logique qui nous échappe, celle qui à la fin de la quête permettra la destruction de l'anneau malgré Frodo. C'est cette conception même qui brouille la distinction entre personnage principal et second couteau et qui, dans le cas de cette scène, invaliderait l'idée que Sam deviendrait le héros du roman en devenant le porteur de l'Anneau. La différence entre personnage principal et secondaire pourrait-elle revêtir une autre forme, non actantielle pourrait-on dire?

La piste que je voudrais suivre commence avec la mention en apparence incongrue de la "chanson " que pourrait devenir la mort tragique de Sam. Le personnage se projette ici dans un avenir où ses actions deviendraient un sujet de poème. Dans le chapitre 8 du même livre IV ("The Stairs of Cirith Ungol »), une telle idée était déjà présente, dans les paroles qu’il échangeait avec Frodo :

"I wonder if we shall ever be put into songs or tales. We're in one, of course; but I mean: put into words, you know, told by the fireside, or read out of a great big book with red and black letters, years and years afterwards. And people will say : "Let's hear about Frodo and the Ring!" And they'll say: "Yes, that's one of my favourite stories. Frodo was very brave, wasn't he, dad?" "Yes, my boy, the famousest of the hobbits, and that's saying a lot." "It's saying a lot too much", said Frodo, and he laughed, a long clear laugh from his heart. (...) "Why, Sam", he said, "to hear you somehow makes me as merry as if the story was already written. But you've left out one of the chief characters: Samwise the stouthearted." "I want to hear more about Sam, dad. Why didn't they put more of his talk, dad? That's what I like, it makes me laugh". And Frodo wouldn't have gone far without Sam, would he, dad? »

Ce passage, par la conscience manifestée par Sam d'être "dans » une histoire, correspond à la dimension métafictionnelle des romans de Tolkien ${ }^{16}$. Mais ces paroles manifestent quelque chose de plus qu'une simple mise en relief de la nature narrative du roman. Sam envisage le moment où, dans un avenir indéterminé, ce que les deux hobbits sont en train de vivre sera raconté au coin du feu et où le public commentera leur histoire. Mais, par les mots de Frodo, un basculement temporel et logique se produit : " to hear you somehow makes me as merry as if the story was already written " : ce qui était imaginé par Sam

16 Étudiée notamment dans deux articles récents : Bowman, Mary R., "The story was already written: narrative theory in The Lord of the Rings" (Narrative, vol. 14, $\mathrm{n}^{\circ} 3$, octobre 2006, p. 272-293) et Brljak, Vladimir, "The books of lost tales: Tolkien as metafictionist " (Tolkien Studies, vol. 7, 2010, p. 1-34). 
comme une prolepse renvoyant à un avenir indéterminé apparaît concrètement dans le présent du récit lui-même, par le rire et les mots de son maître. Frodo fait même mine de presser Sam de demandes semblables à celles des enfants de ce conteur futur et, par cet étrange phénomène, Sam devient lui-même ce conteur, le narrateur de sa propre aventure, au moment même où il est en train de la vivre ${ }^{17}$. Par cette étrange conflagration, l'aventure présente se dédouble : elle serait à la fois une action et, déjà, le récit qui en rend compte. Ce serait un sens possible de la phrase de Sam : "We're in one, of course. " Il en résulte ce que Verlyn Flieger a décrit à propos de cette scène comme " an image of postmodern indeterminacy ", les lecteurs ne se trouvant " neither wholly in the narrative (for we have been reminded that we are reading a book) nor wholly outside it (for as long as we are reading it, the book we are reading has not yet been finished). ${ }^{18}$ Une même indétermination affecte Sam et Frodo, ni totalement dans l'histoire, ni totalement à l'extérieur. Leur différence n'est plus celle d'un sujet et d'un adjuvant, mais celle qui sépare un conteur de son public, mais aussi de son héros puisque, dans le titre du récit envisagé (et conté !) par Sam, seul le nom de Frodo apparait : "Let's hear about Frodo and the Ring! " Ici, Frodo apparaitrait comme sujet non pas parce que ses actions le caractériseraient ainsi, mais parce que Sam, en tant que narrateur, l'a institué comme tel.

Cette conception de Sam pourrait être mise en lien avec son goût pour les chansons et les légendes. Dès le premier chapitre, son père dit de lui : "Crazy about stories of the old days he is, and he listens to all Mr. Bilbo's tales. " Deux passages essentiels peuvent être réinterprétés dans cette perspective. J'ai indiqué précédemment que Sam n'avait pas fait le choix de partir avec Frodo : parce qu'il l'a surpris écoutant à la fenêtre, c'est Gandalf qui lui a imposé cette " punition ». Mais si Sam a commencé à tendre l'oreille au discours du magicien, c'est justement à cause de son goût pour les histoires : " "Don't be a fool! What have you heard, and why did you listen?" (...) "Well, sir," said Sam dithering a little. "I heard a deal that I didn't rightly understand, about an enemy, and rings, and Mr. Bilbo, sir, and dragons, and fiery mountains, and -and Elves, sir. I listened because I couldn't help myself, if you know what I mean. Lor bless me, sir, but I do love tales of that sort. And I believe them too, whatever Ted may say." "

17 Dans cette étrange situation d'énonciation, il apparaît aussi curieusement comme le père de Frodo.

18 Flieger, Verlyn, "A postmodern medievalist? ", in Tolkien's modern Middle Ages, édité par Jane Chance et Alfred K. Sievers, Palgrave Macmillan, 2005, nouvelle impression en 2009, p. 17-28 (p. 24-25). 
Qu'en est-il maintenant de la place de la " chanson » imaginée par Sam au sujet de sa mort héroïque dans sa décision finale de courir défendre le corps de son maître plutôt que de poursuivre la Quête ? Peut-être pourrait-elle être mise en relation avec la " nature " et le " cœur " que Sam jugeait plus fiables que "la tête ". Contre une prise de décision qui ferait intervenir des arguments rationnels comme l'intérêt du monde passant avant celui d'un individu seul, Sam privilégierait une autre façon de diriger sa vie : un choix juste serait celui qui pourrait faire une belle chanson, et ce, même si un tel choix conduisait à un anéantissement général. Mary R. Bowmann a montré comment cette façon de décider guidait les actions d'autres personnages du roman : ainsi, en V, 3, Merry supplie le roi du Rohan de le laisser l'accompagner parce que «I would not have it said of me in song only that I was always left behind. ${ }^{19}$ Mais Sam, contrairement à Merry, aurait la possibilité de devenir lui-même un conteur ou un chanteur de récits.

Ce n'est sans doute pas un hasard si Sam apparait comme un conteur alors que tel n'est pas le cas de Frodo. Dès le deuxième chapitre de l'œuvre, Sam " chante à demi " les mots par lesquels il évoque le départ des elfes loin des rivages de la Terre du Milieu (I, 2). De même, au chapitre 12 du premier livre, Sam entonne une de ses créations, relatant, dans un style de conte folklorique, la rencontre d'un dénommé Tom avec un troll ${ }^{20}$. Au contraire, de Frodo il est dit que " He was seldom moved to make song or rhyme " (II, 7). La seule création dont il soit l'auteur est le poème en hommage à Gandalf, présumé mort dans la Moria, et Frodo préfere laisser à Sam le soin de le compléter, en écrivant un couplet sur les feux d'artifice ${ }^{21}$. En tant que héros, Frodo aurait une activité d'écriture différente, celle, comme Bilbo avant lui, de compiler dans un livre les informations recueillies au cours de ses voyages : les textes qu'il écrit sont des "mémoires " au sens le plus technique du terme et non des contes, chansons ou récits. Le titre des volumes que Frodo remet à Sam au moment de son départ est ainsi intitulé " THE DOWNFALL OF THE LORD OF THE RINGS AND THE RETURN OF THE KING (as seen by the Little People; being the memoirs of Bilbo and Frodo of the Shire, supplemented by the accounts of their friends and the learning of the Wise) »(VI, 9). Cette œuvre appartient au dispositif de métafiction extrêmement complexe mis en place par Tolkien et analysé en détails par Vladimir Brljak. ${ }^{22}$ Dans le prologue de l'œuvre, l'auteur décrit l'histoire complexe des quatre volumes de mémoires écrits par Bilbo et Frodo, auxquels furent ajoutés au cours du temps d'autres textes, tels que « commentaries, generalogies, and various other matters concerning the hobbit members

19 Bowmann, Mary R., art. cit., p. 278.

20 En ce sens, Sam pourrait être, comme cela est suggéré dans la citation de Tolkien mentionnée au début de ce travail, le successeur (encore maladroit) de Bilbo, dont il est justement dit qu'il " écrivait de la poésie» (" he wrote poetry» I, 11).

21 II, 7, p. 351.

22 Brljak, Vladimir, art. cit. 
of the Fellowship ». Cet ensemble constitue " the Red Book of Westmarch ", existant en Terre du Milieu sous la forme de copies très différentes les unes des autres. Ce livre est décrit comme " the most important source for the history of the War of the Ring ", ce qui donne l'impression qu'il constitue la source du Seigneur des Anneaux, voire que Le Seigneur des Anneaux serait une traduction en anglais de ce texte. Or, les noms " memoirs", " accounts " et "learning " qui apparaissent dans le titre du volume écrit par Bilbo et Frodo ne correspondent pas à une forme narrative telle que celle du roman du Tolkien ${ }^{23}$. Entre les deux, une étape essentielle a dû se produire, qui n'apparait pas dans l'histoire du texte telle qu'elle est présentée dans le "prologue " : " the shift to third-person narration, addition of dialogue and various other narrative detail, careful handling of the plot, and so forth -anything, in short, that would be involved in the literarization of a non- or at best semi-literary text $»^{24}$, autrement dit, le passage des mémoires à un conte. Vladimir Brljak suggère de voir dans cette faille de l'histoire du texte -faille selon lui consciemment mise en place par Tolkien- un moyen de miner l'authenticité du texte ${ }^{25}$ et ainsi de mettre en place un univers imaginaire, tout en empêchant d'y croire totalement ${ }^{26}$.

Dans le cadre d'une étude sur le personnage de Sam, il serait possible de proposer une autre solution au problème. Présenté de façon proleptique en IV, 8 comme le conteur de l'histoire de Frodo, Sam pourrait être le chaînon manquant entre le "Red Book of Westmarch » et Le Seigneur des Anneaux de Tolkien, celui qui aurait opéré la transformation décisive des mémoires épars en une narration. C'est ce qui semble apparaître au début du chapitre qui avait été envisagé par Tolkien comme épilogue du Seigneur des Anneaux ${ }^{27}$ : «And one evening in March Master Samwise Gamgee was taking his ease by a fire in his study, and the children were all gathered about him, as was not at all unusual, though it was always supposed to be a special treat. He had been reading aloud (as was usual) from a big Red Book $»^{28}$. Tolkien mentionnait dans le prologue l'existence de plusieurs exemplaires du Livre Rouge, présentant chacun un texte différent. Celui dans lequel Sam lit à voix haute pour ses enfants pourrait être une de ces versions, la version décisive, celle où Sam aurait recomposé les mémoires de ses amis pour en faire l'histoire de "Frodo et de l'anneau ».

23 Brljak, Vladimir, art. cit., p. 11.

24 Brljak, Vladimir, art. cit., p. 12.

25 Brljak, Vladimir, art. cit., p. 16.

26 Brljak, Vladimir, art. cit., p. 23.

27 Supprimé sur les conseils des premiers éditeurs, il a depuis été publié dans Sauron Defeated, un ensemble de textes de Tolkien rassemblés par son fils Christopher (HarperCollinsPublishers, 1992).

28 Sauron Defeated, p. 114. Dans la suite de la scène les enfants de Sam l'assaillent de questions assez semblables à celles qu'il imaginait dans sa conversation avec Frodo dans les escaliers de Cirith Ungol. 
Ainsi, ce ne serait pas leur statut actanciel différent par rapport à la Quête du roman qui ferait de Frodo un héros et de Sam un second couteau. La différence décisive se situerait à un autre niveau, le rapport à l'action en tant que récit en puissance : celui que parcourent les lecteurs du Seigneur des Anneaux. Parce que Frodo est le héros, il est plongé dans les événements sans posséder une telle conscience. Au contraire, Sam sait que ces exploits et ces revers de fortune, ces malheurs et ces joies inattendues, seront un jour racontés. Il est celui qui, peutêtre, finit par les transformer en récit, celui en tout cas qui, dans les escaliers de Cirith Ungol, est déjà par avance leur narrateur. Sam est un personnage secondaire parce qu'il sera - et est déjà - autre chose qu’un personnage.

Antoine Paris

Université Paris IV - Sorbonne /

Université de Montréal antoine7.paris@wanadoo.fr

\title{
Résumé
}

Subordonné à Frodo par le schéma actantiel du Seigneur des Anneaux et par son statut social qui le rattache à un autre registre ou genre littéraire, Sam peut aussi être vu comme l'image d'un serviteur de la Première guerre mondiale, tendrement fidèle à son officier. Une importance particulière sera donnée au chapitre final du livre IV, dans lequel Sam pourrait devenir le héros du roman, mais le refuse. Cet épisode manifeste qu'il est un personnage secondaire parce qu'il a conscience d'être dans un récit; parce qu'il en est, en puissance, déjà un narrateur.

\section{Mots-clés}

Faire-valoir, Frodo, Tolkien.

\begin{abstract}
Sam is subordinate to Frodo because of the actantial model of the Lord of the Rings and of his social status, which makes him related to another register or literary genre. Sam may also be seen as similar to a First World War "batman", tenderly faithful to his officer. Close attention will be paid to the final chapter of book IV, in which Sam could become the hero of the novel, but refuses to do so. This episode shows Sam as a sidekick because he is aware of being in a story; because potentially he is already its narrator.
\end{abstract}

Keywords

Sidekicks, Frodo, Tolkien. 